Cinémas

Revue d'études cinématographiques

Journal of Film Studies

\title{
Du scénario de film aux scénarios multimédias dits interactifs : étude des pratiques d'écriture scénaristique
}

\section{Isabelle Raynauld}

Volume 9, numéro 2-3, printemps 1999

Les Scénarios fictifs

URI : https://id.erudit.org/iderudit/024791ar

DOI : https://doi.org/10.7202/024791ar

Aller au sommaire du numéro

Éditeur(s)

Cinémas

ISSN

1181-6945 (imprimé)

1705-6500 (numérique)

Découvrir la revue

Citer cet article

Raynauld, I. (1999). Du scénario de film aux scénarios multimédias dits interactifs : étude des pratiques d'écriture scénaristique. Cinémas, 9(2-3),

147-156. https://doi.org/10.7202/024791ar
Résumé de l'article

Penser et écrire pour le multimédia. La scénarisation destinée aux fictions sur CD-ROM est-elle en train de développer des règles d'écriture très différentes de celles de la scénarisation cinématographique? Réputées pour la capacité à proposer des structures textuelles " éclatées ", les fictions multimédiatiques sont-elles vraiment interactives et non linéaires? Jusqu'à quel point les règles d'écriture du scénario de film sont-elles valables et adaptables aux fictions dites interactives? Le multimédia a-t-il vraiment transformé notre façon de raconter des histoires? Enfin, que change cette nouvelle technologie à notre façon de lire, d'écrire et d'expérimenter un récit? 


\title{
Du scénario de film aux scénarios multimédias dits interactifs : étude des pratiques d'écriture scénaristique
}

\section{Isabelle Raynauld}

\section{RÉSUMÉ}

Penser et écrire pour le multimédia. La scénarisation destinée aux fictions sur CD-ROM est-elle en train de développer des règles d'écriture très différentes de celles de la scénarisation cinématographique? Réputées pour la capacité à proposer des structures textuelles "éclatées", les fictions multimédiatiques sont-elles vraiment interactives et non linéaires? Jusqu'à quel point les règles d'écriture du scénario de film sont-elles valables et adaptables aux fictions dites interactives? Le multimédia a-t-il vraiment transformé notre façon de raconter des histoires? Enfin, que change cette nouvelle technologie à notre façon de lire, d'écrire et d'expérimenter un récit?

\begin{abstract}
What is writing for multimedia? This arricle questions the developments of screenwriting rules and functions in regard to multimedia technologies. How does the narrative structure of a fiction offered on a CD-ROM change and adapt to an interactive and non-linear environment? How interactive and non-linear is it and what can we do with it? Do electronic media really transform the way we tell stories? What happens to reading strategies and dramatic impact when the script reader has become a hypertext navigator? What does the hypertext technology do to the user's involvement
\end{abstract}


in the fiction? And finally, how do we write a script for multimedia entertainment and what questions does that "new practice" raise for the theory and the future of screenwriting?

Penser et écrire pour le multimédia. Jusqu’à quel point cette "nouvelle pratique» change-t-elle les règles du jeu, de l'écriture? Multimédia, interactivité, arborescence, non-linéarité, lecteurutilisateur-créateur de récit, système de navigation... autant d'expressions qui ont envahi notre univers du jour au lendemain. Notre méconnaissance à leur égard apparaît comme l'aveu d'une résistance au "progrès", la preuve de notre enfermement dans la "linéarité " des scénarios de films. Car les adeptes du multimédia parlent, eux, de "révolution", affichant par moment une croyance inébranlable, celle justement d'avoir inventé la non-linéarité.

Nous nous intéressons au multimédia parce que cette "nouvelle» technologie pose des questions et bouleverse les domaines de la théorie et de la pratique du scénario. Plus précisément, ces nouvelles technologies inaugurent un problème qui pourrait s'énoncer comme suit: en quoi un environnement entendre aussi document - multimédiatique transforme-t-il nos pratiques d'écriture et de lecture?

Notre approche est celle des historiens du cinéma, des scénaristes et de passionnés de la théorie du texte scénaristique. Bien sûr, ces différentes approches ont chacune leur champ de pertinence en regard du problème posé, mais leur croisement permet de soulever et de traverser des questions variées. En voici, d'entrée de jeu, quelques-unes: à quel point les fictions multimédiatiques sont-elles vraiment interactives et non linéaires? à quel point les règles d'écriture du scénario de film sont-elles valables et adaptables aux fictions dites interactives? le multimédia a-t-il vraiment transformé notre façon de raconter des histoires? cette technologie oblige-t-elle des changements dans nos façons de lire, d'écrire et d'expérimenter un récit?

Afin d'explorer ces questions, nous nous intéresserons au multimédia en suivant trois axes d'analyse:

1) l'invention technologique d'un point de vue historique: qu'est-ce que la nouveauté? En quoi cette dite "nouvelle 
pratique d'écriture" est-elle plus "révolutionnaire»; à l'aune de la courte histoire du cinéma, en quoi a-t-elle plus d'impact sur la structuration de la fiction, que, par exemple, les développements des principes du montage cinématographiques?

2) le lecteur comme acteur et/ou auteur: comment cette fameuse "interactivité" influence-t-elle notre compréhension de Thistoire et quadvient-il du processus d'identification du lecteurspectateur dans une fiction qu'il construit supposément lui-même?

3) dramaturgie multimédiatique: participe-t-on vraiment, en ce moment, à l'émergence d'une "nouvelle écriture», en termes de structuration de l'histoire?

\section{L'invention technologique d'un point de vue historique}

Quelle place peuvent avoir les mots "nouveauté" et "invention" en histoire du cinéma? Le cinéma n’en est pas à sa première invention ni à sa première innovation technologique. Pourtant, à chaque fois, plus d'un sceptique a cru que le cinéma "classique " ne s'en remettrait pas, qu'il succomberait après chaque innovation, qu'il s'agisse des développements du montage, de l'augmentation du métrage des films, de la mobilité accrue de la caméra, de l'introduction du son et des dialogues dans toutes les langues, de l'arrivée de la couleur, des pellicules grand format et des projections en trois dimensions (3D), etc. $A$ chaque fois, un critique a déclaré le plus sérieusement du monde que le cinéma était en péril. Lors du passage du muet au parlant, combien nombreuses furent les mises en garde et les résistances contre la tentation de faire des films parlants, plusieurs annonçant même la contamination de l'image par le son!

Si chaque nouveauté ou invention donne au créateur le sentiment euphorique d'un pouvoir renouvelé - d'une transformation radicale, d'un "progrès» face à l'ancien - la nouveauté effraie et menace pourtant tout autant qu'elle n'attire. Si le cinéma a lui-même souvent été présenté en état de crise, son avènement au début du siècle a jadis inquiété un autre dispositif, le milieu du théâtre, qui s'était senti alors menacé dans ses lieux et son répertoire.

Ce que nous apprennent ces réactions radicales face "aux nouvelles technologies", c'est qu'à chaque fois, artistes, critiques 
et intellectuels doivent redéfinir ce qui fait la spécificité de leur pratique, de leur art. En ce moment, il est fascinant de constater combien les réactions face au multimédia et aux fictions interactives sur CD-ROM ressemblent aux jugements propagés, aux débuts du cinéma, sur l'écriture des scénarios: on critiquait les maladresses de la manière dite cinématographique de "raconter une histoire" par rapport à l'aisance et à la souplesse de l'écriture romanesque.

Parallèlement à ces commentaires parfois fondés, souvent désobligeants, on a vu se développer, dès les années 1910, une technique d'écriture de scénarios dont les principes de base sont étonnamment semblables aux supposés nouveaux procédés d'écriture interactive et multimédiatique.

À titre d'exemples, voici quelques-uns de ces préceptes, datés respectivement de 1911 et de 1996:

Photoplay, in a word, is nor an adaptation of another branch of literary work, but is possessed of a technique all its own. There are, of course, the broad basic rules of literary construction and dramatic development, applicable to all forms of literature, wether written or verbally expressed, but in the past few years the art of the photoplays has become possessed of a technique that is applicable only to writing of picture plays and to no other form (Sargent, p. 41).

Multimedia refers to an environment. The distinctive characteristic of multimedia is interactivity. It is the mechanism that allows you to more between the various media. The fact of the media itself is not interesting (Blow away what preceded you). The point is, if you're going to be scripting multimedia experiences, be mindful of the intense personal level on which your audience will be participating. The first lesson of this book is to never forget your audience (Varchol, p. 43).

Encore en 1911:

"Every art form strives for a sense of unity". A script works as a whole [...]. It means constructing your story in a way that will give it form, focus, momentum, clarity. It means finding ways to help your audience 
"get with" your story, and involving your audience all along the way. It means crafting your story into dramatic form. Since this action is dramatic form. Since this action is dramatic and visual (not expressed through dialogue), it pushes the story forward (Sargent, p. 88).

Et en 1996, voici l'objet de discussion sur un site consacré à l'écriture entre "écrivains interactifs" du WWWeb: "The key is to involve the audience (user) emotionally. How do we do that in an interactive world?» M. Utvitch (écrivain interactif, selon l'expression américaine interactive writer): "Involve them emotionally, involve them actually. Make them do things, solve problems, puzzles, respond to situations using their own judgement."

De 1911 à 1996, quels "progrès" a-t-on vus naître en termes de constructions narratives? Quels sont les points communs entre ces deux époques dites novatrices et même révolutionnaires sur le plan technologique? Quelle est la spécificité d'une pratique face à une autre? Et en quoi la participation du spectateur et son engagement dans la fiction diffèrent-ils? Sans vouloir donner des réponses forcément trop brèves à toutes ces questions, leur énumération résume néanmoins les préoccupations qui structurent notre second point.

\section{Comment est construite l'identification du spectateur dans un document interactif?}

Pour traiter des notions d'identification du spectateur et de construction, dans le texte scénaristique, des attentes et des expectatives de ce dernier, nous nous appuyons essentiellement sur les travaux d'Umberto Eco, de Roland Barthes, de Christian Metz, de Hans Robert Jauss, de Gérard Genette et de Roger Odin. Transposées et adaptées à l'étude du texte scénaristique (de cinéma et de multimédia), la théorie littéraire et la sémiologie s'avèrent des outils extrêmement utiles pour comprendre et différencier des pratiques d'écriture dites "techniques». Notre approche vise à poursuivre notre étude du scénario comme texte tout en analysant en quoi écrire un scénario destiné à une fiction sur support CD-ROM est différente ou non de la scénarisation cinématographique. 


\section{- LE LECTEUR COMME AUTEUR ET / OU ACTEUR}

Alors que le cinéma classique vante la transparence du montage qui permet de donner l'illusion que l'histoire se raconte d'elle-même, les promoteurs de fictions interactives vendent l'illusion que le lecteur est son propre auteur, qu'il est celui qui, en quelque sorte, raconte l'histoire. Pourtant, voici ce qu'un scénariste interactif écrit :

The life of a screenwriter of an interactive film much better than the life of a Hollywood writer because by making an interactive script, you build a better mousetrap, you become indispensable. Because you know how it all fits together long before the rest of them do (Michael Kaplan, cité par Varchol, p. 3).

Vive la liberté de choix! En fait, qu'est-ce au juste qu'une fiction interactive? À y regarder de plus près, plusieurs caractéristiques alarmantes pour les adeptes de nouveautés surgissent: la fiction sur CD-ROM est beaucoup plus "hyper sélective " " qu'interactive. L'interactivité est en fait la réalisation d'un ensemble de choix prévus, testés et tracés d'avance. La fiction interactive est construite pour vous impliquer et vous faire croire qu'il s'agit d'une histoire faite uniquement pour vous. Voici comment est écrit le prologue de la célèbre fiction sur CDROM Myst:

Je réalisai à l'instant même où je tombais dans la crevasse que le livre ne serait pas détruit comme je l'avais prévu. Il continuait de tomber dans cette étendue étoilée dont je n'ai vu qu'une image fugace. J'ai tenté d'imaginer où il avait pu atterrir. Je dois cependant avouer que de telles interrogations sont vaines. Toujours est-il que je ne cesse de me demander dans quelles mains mon livre Myst finira un jour par tomber. Je sais que mes craintes ne seront peut-être jamais apaisées. C'est pourquoi j'arrête, réalisant que la fin n'a peut-être pas encore été écrite (Miller, Rand et Robyn, 1995).

Soulignons, au passage, la parenté indéniable de ce texte avec le procédé d'introduction, bien connu en littérature, du "livre trouvé » et du "texte qui reste toujours et encore à écrire" (Sterne, 1940). 
Dans l'univers des "fictions multimédias", chacun sait que malgré des choix multiples, on nous interdit l'accès au dénouement final. La curiosité est punie. L'information fondamentale - le cœur du récit - ne nous est accessible que selon un cheminement précis. Si on déroge à ce trajet tracé d'avance, à force d'astuces ou en se croyant plus rapide qu'un autre à résoudre les énigmes du récit, on nous renvoie à la case départ (un peu comme dans le Jeu de l'oie, en fait!) La supposée structure en arborescence est, en fait, composée à la fois d'une structure qui est profondément linéaire et qui répond, malgré les apparences, aux impératifs du récit classique (exposition, développement, dénouement) et d'une structure dégressive (à la manière d'un entonnoir), mais qui est simultanément nourrie de digressions multiples (clairières d'actions, histoires secondaires, fausses pistes) qui retardent, détournent et surtout occupent le lecteur durant une quarantaine d'heures.

C'est que l'interactivité n'a pas inventé la non-linéarité. Au contraire, c'est la linéarité qui fait partie de la conception d'un document interactif, alors qu'il existe des récits littéraires, filmiques et picturaux non linéaires depuis les tous débuts de l'histoire de la civilisation. De plus, d'un point de vue théorique, une interactivité "réelle" a comme base définitionnelle une action réciproque; elle est en principe une activité qui influence, qui fait changer l'histoire. Mais dans les CD-ROM de fiction, l'histoire interactive est "déjà cartographiée ". Son moteur de conception est le procédé de causalité du si $A$, alors $B$. Ce raisonnement logique est un processus linéaire en soi, l'élément $B$ ne pouvant apparaître avant le $A$. Aussi, il est clair que les attentes du lecteur-utilisateur "idéal" (Eco, 1985) tel qu'imaginé par le scénariste correspondent à certaines règles de la dramaturgie, mais surtout à celles de la logique classique qui veut entre autres que si $A$ est égal à $B$ et que $B$ est égal à $C$, alors $A$ est égal à $C$. La structure en toile d'araignée n'engloutit donc pas l'histoire mais bien l'utilisateur en lui donnant, par exemple, cette fausse impression qu'il peut se perdre.

Dans cette perspective, en quoi l'expérience de lecture - la navigation interactive - se distingue-t-elle de l'expérience de visionnement d'un film? Les deux expériences sont évidemment très différentes et ceci nous amène à notre dernier point. 


\section{Nouvelle écriture ou nouvelle lecture?}

La différence principale entre l'écriture scénaristique destinée au cinéma et celle dite interactive réside selon nous beaucoup plus dans la lecture du texte ("lecture du texte" scénaristique et filmique entendue au sens barthien) que dans l'écriture. Comme on l'a vu, les principes d'écriture changent beaucoup moins qu'on aurait d'abord pu l'imaginer et, en termes dramaturgiques, le multimédia est encore très loin d'une révolution. C'est plutôt la posture de lecture et d'appréhension du texte qui, du film au CD-ROM de fiction, se transforme. On passe du "regarder au faire" ou, comme l'aurait formulé Greimas, au "faire faire». Si le scénario de film prévoit les réactions et les attentes du spectateur "idéal ", le scénario de multimédia prévoit en plus le passage à l'acte du lecteur-utilisateur. En effet, face à une fiction dite interactive sur CD-ROM, le lecteur doit littéralement avancer et naviguer dans le récit afin que l'histoire lui soit racontée. L'histoire, loin de se raconter toute seule, exige d'être racontée par le lecteur, à force de déductions et d'inductions. Et pour ce faire, le lecteur doit avoir un contact réel avec la machine qui, elle, donne accès au médium, au dispositif ${ }^{2}$. La vieille question de la passivité du spectateur de film est de nouveau d'actualité. Mais pour pouvoir lire et donc recevoir l'histoire, que doit faire exactement le lecteur-utilisateur de CDROM? Il explore, il découvre, il accumule de l'information. Surtout, il apprend les règles du jeu et il apprivoise le fonctionnement interne de "l'environnement" proposé (Cotton et Olivier, 1992). Connaître les règles de l'univers permet au lecteur-utilisateur d'aller plus loin, plus vite et d'avancer dans le récit. Tout se passe comme si le lecteur devait avec chaque nouvelle fiction sur CD-ROM apprendre à lire pour avoir accès au récit. La structure narrative intègre les règles du jeu et s'en sert comme moteur de l'histoire.

\section{- L'IDENTIFICATION DU SPECTATEUR PRISE DEUX}

Mais quarrive-t-il au processus d'identification du lecteur? S'il passe à l'acte, le lecteur interactif continue tout de même à anticiper, à lier des séquences pour comprendre l'histoire, à être curieux, etc. Ce qui change est l'expérience du "passage du 
temps» et donc l'expérience de la catharsis. Les usagers déçus par le dénouement d'une fiction interactive sont fort nombreux! C'est que l'effort de lecture investit ne vaut pas, semble-t-il pour plusieurs, la découverte du point final. À la différence d'un roman bien écrit et d'un film bien tourné, le lecteur-utilisateur se sent floué parce qu'il n'a ni écrit, en mode réellement interactif, une histoire tel que promis en début de jeu puisque, comme on l'a vu, il n'y a pas de véritable interactivité ni ne s'est abandonné à une histoire "comme si elle se racontait toute seule», car il a dû jouer - en fait travailler -- à résoudre les éléments du récit afin d'en connaître le dénouement. Finalement, on voit que ce que ces scénarios ont à offrir de réellement différent est le concept d'un lecteur "utilisateur». Si le scénario cinématographique s'adresse à un lecteur créateur qui fera du texte un film, il s'adresse aussi à un lecteur spectateur qui verra ou a vu le film. Le scénario interactif, lui, est structuré en fonction des interventions actives du lecteur face au texte, c'est-à-dire en fonction de l'usage qu'il en fera et du parcours qu'il devra, bon gré mal gré, suivre s'il veut atteindre "la fin de l'histoire».

En conclusion, il faut selon nous différencier la "narrativité interactive" (structure linéaire dégressive) des «zones d'interactivité" de l'écriture interactive (conception qui prévoit les liens et trace une cartographie de l'arborescence) de la "navigation interactive" (où le lecteur explore et découvre le document ${ }^{3}$ ).

Si chaque fiction narrative est bien construite avec une série de règles - comme un jeu —, il faut tout de même admettre, qu'on soit de tendance "révolutionnaire» ou non, que c'est plutôt à l'Antiquité qu'on doit nos grands principes dramaturgiques actuels. Pour nous, c'est la façon dont le lecteur/ utilisateur/ spectateur "entre" dans la fiction et "y croit ou non" qui fait la vraie différence entre une pratique d'écriture scénaristique et une autre.

\section{Université de Montréal}

NOTES

1 L'expression est de P. C. Bélanger (Le Système Vidéoway. Modalités d'adoption d'un système interactif de télévision (thèse de doctorat). Montréal: Université de Montréal, 1991). 
2 Le rapport de l'homme à la machine est le concept fondateur de toutes les recherches entreprises en théorie de l'informatique et de la communication depuis 35 ans et plus. Voir notamment les travaux de Nicholas Négroponte du M.I.T., L'Homme numérique (Paris: Robert Laffont, 1995).

3 Ces concepts et distinctions sont à approfondir et feront l'objet d'une communication présentée au premier colloque international du Centre de recherche sur l'intermédialité (CRI) de l'Université de Montréal (sous la direction d'André Gaudreault et de Terry Cochran), Musée d'art contemporain, du 2 au 6 mars 1999.

\section{OUVRAGES CITÉS}

Barthes, Roland. Plaisir du texte. Paris: Seuil, 1973.

Bélanger, P. C. Le Système Vidéoway. Modalités d'adoption d'un système interactif de télévision (thèse de doctorat). Montréal: Université de Montréal, 1991.

Cotton B. et Olivier, R. Understanding Hypermedia. From multimedia to virtual reality. London: Phaidon Press, 1992.

Eco, Umberto. Lector in Fabula. Paris: Grasset, 1985.

Garand, Timothy. Writing for Multimedia. Newton : Focal Press, 1997.

Genette, Gérard. Figures III. Paris: Seuil, 1972.

Genette, Gérard. Nouveau Discours du récit. Paris: Seuil, 1983.

Greimas, A. et J. Courtée. Sémiotique. Tomes 1 et 2. Paris: Hachette, 1979.

Jauss, Hans Robert. Pour une esthétique de la réception. Paris: Gallimard, 1978.

Metz, Christian. L'Énonciation impersonnelle ou le site du film. Paris: MéridiensKlincksieck, 1991.

Miller, Rand et Robyn. Myst. Cleveland: Broderbund Software Inc. et Cyan Inc., 1995.

Negroponte, Nicholas. L'Homme numérique. Paris : Robert Laffont, 1995.

Odin, Roger. «Du spectateur fictionnalisant au nouveau spectateur: approche sémiopragmatique». Iris, n" 8 (1988), p. 121-139.

Odin, Roger. Cinéma et production de sens. Paris: Armand Colin, 1990.

Sargent, Epes Winthrop. The Technique of the Photoplay. New York: The Moving Picture World, 1911.

Seger, Linda. Making a Good Script Great. New Yord: Dodd, Mead \& Company, 1987.

Sterne, Laurence. The Life and Opinions of Tristram Shandy, Gentleman. Indianapolis: The Bobbs Merrill Co., 1940.

Varchol, Douglas J. The Multimedia Scriptwriting Workshop. Alameda : Sybex, 1996.

Wimberley, Darryl et Jon Samsel. Interactive Writers Handbook. San Francisco: Caronnade Group, 1996. 